\title{
Biomarkers of early respiratory effects in smoking adolescents
}

\author{
E. Van Miert, A. Sardella and A. Bernard
}

\begin{abstract}
Noninvasive biomarkers can be used to evaluate airways damage caused by tobacco smoke, but studies so far have only involved adult smokers. In this study, we evaluated whether such biomarkers can detect early respiratory effects in adolescents passively or actively exposed to tobacco smoke.
\end{abstract}

In a cross-sectional study of $\mathbf{8 4 5}$ adolescents (mean age 16 yrs), we measured exhaled nitric oxide (NO) and various epithelial markers in nasal lavage fluid (NALF) and serum, including Clara cell protein (CC16) and surfactant protein (SP)-D. Information about smoking habits and potential confounders was collected by questionnaire. Four groups of equal size $(n=36)$, of nonsmokers, passive smokers, light smokers ( $<5$ cigarettes $\cdot$ day $^{-1}$, median 0.08 pack-yrs) and heavy smokers ( $\geqslant 5$ cigarettes $\cdot$ day $^{-1}$, median 0.35 pack-yrs), were matched using an automated procedure.

The levels of exhaled NO and of CC16 in NALF were significantly decreased in the group of heavy smokers. A trend towards lower levels of CC16 in NALF was observed in passive smokers. There were no significant changes in serum CC16 and SP-D, which suggests that the deep lung epithelium had not yet been affected by smoking.

In conclusion, tobacco smoke can cause early changes in the airways of adolescents with a cumulative smoking history of $<1$ pack-yr.

KEYWORDS: Adolescents, biomarkers, cigarette smoke, Clara cell protein 16, nasal lavage fluid, nitric oxide

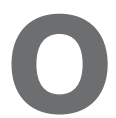
ver recent years, several approaches have become available to assess the response of the respiratory system to inhaled agents. Bio-imaging techniques and functional assays have undergone significant improvements, but progress has also been achieved using noninvasive biomarker methods, e.g. the analysis of exhaled breath, the detection of inflammatory cells and mediators in induced sputum, and the analysis of lung-specific proteins in serum [1-4]. Recently, analysis of biomarkers collected after nasal lavage has also received increased attention [5]. Multiple studies have been published showing changes in biomarker levels in diseased individuals, e.g. increased exhaled nitric oxide (NO) during asthma exacerbations, increased levels of surfactant protein (SP)-D in serum of chronic obstructive pulmonary disease (COPD) patients or decreased levels of Clara cell protein (CC16) in serum or induced sputum of COPD patients [6-8].

One of the most valuable applications of biomarkers is the detection of preclinical phases of disease development. In this respect, asymptomatic smokers are particularly interesting, as they represent a large group of subjects chronically exposed to agents having clear detrimental effects on human health $[9,10]$. Structural and functional changes in the nose have been described in smokers, starting from 10 pack-yrs [11]. Smokers show decreased levels of serum CC16 [12], which can already be detected in young adults with a median of only 3.8 pack-yrs [13]. The reduced levels of serum CC16 in smokers most probably reflect a decrease of CC16 in bronchoalveolar lavage fluid paralleling the progressive loss of CC16-positive cells in terminal airways [14]. The concentrations of SP-A and $-\mathrm{B}$, conversely, rise in the serum of smokers [12] as a consequence of the disruption of epithelial barriers by tobacco smoke. Smokers also have a decreased concentration of $\mathrm{NO}$ in the exhaled breath (mean $-52 \%$, average 10.2 pack-yrs), which correlates with their daily cigarette consumption $(r=0.77, p<0.001)$ [15]. Decreased levels of exhaled NO fraction were also observed in infants exposed pre- and post-natally to cigarette smoke compared with never-exposed infants or infants exposed to tobacco smoke only after birth [16]. In agreement with these biomarker studies, LEDERER et al. [17] recently reported subclinical parenchymal lung disease using computed tomography and spirometric measurements in a generally healthy cohort of older adult smokers.

\section{AFFILIATIONS}

Louvain Centre for Toxicology and Applied Pharmacology, Faculty of Medicine, Catholic University of Louvain, Brussels, Belgium.

CORRESPONDENCE

A. Bernard

Louvain Centre for Toxicology and Applied Pharmacology, Faculty of Medicine

Catholic University of Louvain Avenue E. Mounier 53.02

B-1200 Brussels

Belgium

E-mail: Alfred.Bernard@uclouvain.be

Received:

Jan 042011

Accepted after revision:

April 172011

First published online:

May 122011 
Until now, biomarker studies among smokers have been conducted on adult subjects with a smoking history of at least several pack-years. Here, we investigated whether noninvasive biomarkers of the upper and lower respiratory tract can detect subclinical changes in the airways of adolescents with an active or passive exposure to cigarette smoke.

\section{MATERIAL AND METHODS \\ Study population}

The study protocol was approved by the Ethics Committee of the Faculty of Medicine of the Catholic University of Louvain (Brussels, Belgium) and complied with all applicable requirements of international regulations. Details about the crosssectional study on adolescents in Belgium have been described elsewhere [18]. Briefly, the study was conducted among adolescents in the third and fourth grades of secondary school. A questionnaire and a written agreement to participate in the study were obtained from the adolescents' parents. The questionnaire addressed aspects related to social and medical characteristics of the adolescent and their family, and to the in-house and out-of-house environment.

\section{Examination of study participants}

The adolescents participating in the study were examined in their school. Examination took place between 09:00 and 15:00 h. For each test or sample collection, the time was recorded to adjust for possible diurnal variations in the biomarker levels. The examination consisted of an interview enquiring about the smoking status and recent ( $<12$ months') respiratory symptoms, followed by the measurement of height and weight, exhaled NO levels and lung function parameters, and the collection of a blood and of two nasal lavage fluid (NALF) samples. The smoking status used for grouping the study participants was based on the information provided by the adolescents themselves. The active smokers were divided into "light smokers" and "heavy smokers" depending on the daily cigarette consumption $(<5$ or $\geqslant 5$ cigarettes, respectively). The allocation criterion of 5 cigarettes $\cdot$ day $^{-1}$ was selected for pragmatic reasons, i.e. it allowed definition of a group of heavy smokers of sufficient size and a slightly larger group of light smokers, better enabling the matching procedure (see statistical analyses section). A sample of venous blood was collected in a dry tube, allowed to clot overnight at $4{ }^{\circ} \mathrm{C}$ and then centrifuged at $2,000 \times \mathrm{g}$ for $10 \mathrm{~min}$. Serum was decanted and stored at $-18^{\circ} \mathrm{C}$ until biomarker analysis. NALF samples were collected from both nostrils. Participants were asked to sit down, bend forward and put their heads down. $2.5 \mathrm{~mL}$ of sterile physiological saline at $37^{\circ} \mathrm{C}$ were instilled into each nostril by a disposable tip connected to a peristaltic pump. After $10 \mathrm{~s}$, students were asked to lift their head and the lavage fluid was collected using a small funnel. The NALF samples were stored at $-20^{\circ} \mathrm{C}$ until evaluation. The concentration of $\mathrm{NO}$ in exhaled air was determined with the NIOX ${ }^{\mathrm{TM}}$ analyser (Aerocrine AB, Solna, Sweden) according to the guidelines of the American Thoracic Society [19]. Total and aeroallergen-specific immunoglobulin (Ig)E (house-dust mite, cat epithelium, dog dander, moulds, tree pollen, grass pollen and herbaceous pollen mixture) concentrations in serum were determined using the Immulite $\operatorname{IgE}$ kit (Diagnostic Products Company, Los Angeles, CA, USA). Sensitisation against specific aeroallergens was defined as a serum concentration of specific $\mathrm{IgE}>0.35 \mathrm{kIU} \cdot \mathrm{L}^{-1}$. CC16 in serum and NALF was measured by latex immunoassay using a rabbit
anti-CC16 antibody (Dakopatts, Glostrup, Denmark) and standard CC16 purified in our laboratory at the Louvain Centre for Toxicology and Applied Pharmacology, Faculty of Medicine, Catholic University of Louvain [20]. The serum concentration of SP-D was determined using a commercially available ELISA kit (code no. YSE-7744; Yamasa Corporation, Choshi, Japan). Albumin, creatinine and urea were quantified by the Beckman Synchron CX5 Delta Clinical System (Beckman Coulter Inc., Fullerton, CA, USA). Concentrations of biomarkers in NALF were adjusted for the variable dilution of the recovered epithelial lining fluid, either by calculating the absolute amount of recovered protein or by adjusting the concentration in NALF with the plasma/NALF concentration ratio of urea [21, 22]. These adjustments were made for each nostril separately and then the mean value was calculated and used for the statistical analyses.

\section{Statistical analyses}

Matching procedures and statistical analyses were performed using the $\mathrm{R}$ statistical package (The $\mathrm{R}$ Project, Institute for Statistics and Mathematics, Vienna University of Economics and Business, Vienna, Austria) [23-26]. The matching was performed relative to the group of heavy smokers, which included 36 subjects, except for biomarkers in NALF for which we excluded the adolescents who had a cold $(n=9)$ during the previous 2 weeks. Groups equivalent to the heavy smokers group were generated from the total groups of nonsmokers $(n=507)$, passive smokers $(n=254)$ and light smokers $(n=48)$ using an automated matching procedure ("optmatch package") based on the following characteristics: "sex", "age", "parental allergy or asthma" and "parent with higher education". Associations of the smoking status with the personal and familial characteristics, medical characteristics or antecedents, the self-reported respiratory symptoms, sensitisation and characteristics of the in-house and out-of-house environment were analysed by pair-wise comparison with the nonsmokers group using the Chi-squared test. Comparisons of the continuous variables with the nonsmokers group were performed using one-way ANOVA followed by Dunnett's post hoc test. Models for biomarkers in NALF, blood and exhaled air were built by means of generalised linear modelling using the Gaussian distribution. The initial models were built assessing the association of each biomarker with being a light smoker, a heavy smoker or a passive smoker, with age, sex, body mass index (BMI), birth weight, total serum IgE concentration, time of sample collection, the social and medical characteristics and variables reflecting the in-house and out-of-house environment quality (e.g. siblings, mould on bedroom wall, wood smoke, air fresheners, cleaning with bleach). These models were run by also testing the interactions between sex and the different smoking statuses (passive smoker $\times$ sex, light smoker $\times$ sex, and heavy smoker $x$ sex). For serum markers, we included in the models the serum creatinine concentration to account for the influence of renal function. The obtained models were optimised using a backward stepwise algorithm using the Akaike Information Criterion (AIC; R-function: "step"). For each of the investigated outcomes, the model with the lowest AIC score was retained. Statistical procedures on continuous variables were performed after log-transformation. Graphs and statistics on adjusted concentrations were generated using GraphPad Prism version 5.00 for Windows (GraphPad Software, San Diego, CA, USA). Relationships between biomarkers and 
cigarette consumption were assessed using the Spearman nonparametric correlation test. The threshold for statistical significance was set at $\mathrm{p}<0.05$.

\section{RESULTS}

\section{Characterisation of subpopulations}

An overview of the characteristics of the different subpopulations is given in table 1 . There were no or very few differences between the groups regarding sex, age, BMI and birth weight. As expected from adolescents who started smoking recently, the cumulative cigarette consumption was very low, with a median of 0.08 and 0.35 pack-yrs for the light and heavy smokers, respectively. Despite the matching procedure, a few differences persisted between the groups, in particular concerning the place of residence and the exposure to older siblings. The different groups had similar prevalences of allergic sensitisation or of ever-diagnosed asthma, hay fever and lower respiratory tract infections. The prevalence of allergic rhinitis and frequent cold tended to be higher among heavy smokers. Compared with the nonsmokers, all smoker groups showed a trend towards higher prevalences of upper respiratory symptoms such as cough crisis, nasal problems and wheezing.

\section{Epithelial biomarkers}

Table 2 shows the results of the univariate analyses of biomarkers. The NALF volumes as well as the urea concentrations were not significantly different between the nonsmokers and the other groups. The median CC16 concentration in NALF was decreased in all smoker groups, reaching statistical significance for the heavy smokers group (median $61 \%$ lower than nonsmokers). A similar pattern of significant decreases was found after adjustment for the variable dilution of NALF sample by calculating the

TABLE 1 Overview characteristics of subpopulations

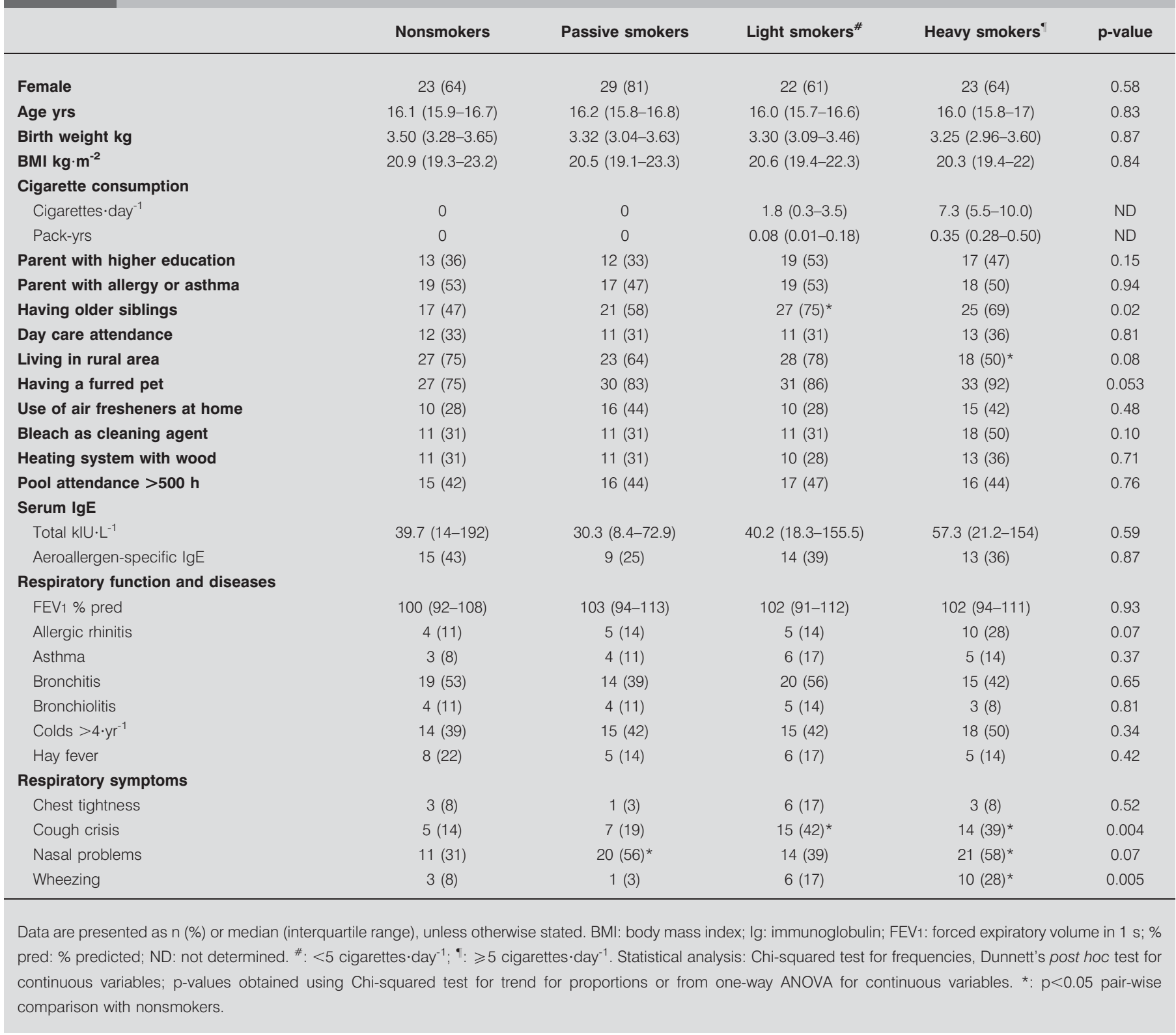


TABLE 2 Biomarkers in nasal lavage fluid (NALF), serum and exhaled breath

\begin{tabular}{|c|c|c|c|c|c|}
\hline & Nonsmokers & Passive smokers & Light smokers ${ }^{\#}$ & Heavy smokers" & p-value \\
\hline \multicolumn{6}{|l|}{ Markers in NALF } \\
\hline \multicolumn{6}{|l|}{ Urea } \\
\hline Concentration $\mathrm{mg} \cdot \mathrm{L}^{-1}$ & $38.8(32-57.7)$ & $41.2(29.3-50.4)$ & $46.8(30.6-62.6)$ & $36.5(28.4-42.6)$ & 0.18 \\
\hline Recovered mg & $0.15(0.11-0.19)$ & $0.15(0.12-0.18)$ & $0.15(0.13-0.21)$ & $0.13(0.11-0.18)$ & 0.33 \\
\hline Concentration $\mu \mathrm{g} \cdot \mathrm{L}^{-1}$ & $28.7(11.5-85.4)$ & $9.5(2.8-50.8)$ & $19.1(9.5-54.2)$ & $11.2(3.6-26.3)^{*}$ & 0.050 \\
\hline Recovered ng & $94(45-313)$ & $41(11-174)$ & $71(37-172)$ & $45(15-100)^{*}$ & 0.04 \\
\hline Adjusted for urea ratio $\mu \mathrm{g} \cdot \mathrm{L}^{-1}$ & $174(101-533)$ & $100(24-307)$ & $135(58-243)$ & $61(24-155)^{\star}$ & 0.02 \\
\hline \multicolumn{6}{|l|}{ Albumin } \\
\hline Concentration $\mathrm{mg} \cdot \mathrm{L}^{-1}$ & $8.8(4.1-16.5)$ & $6.3(3.4-22.7)$ & $9.7(6.1-15.5)$ & $5.8(2.6-13.5)$ & 0.62 \\
\hline \multicolumn{6}{|l|}{ Markers in serum } \\
\hline \multicolumn{6}{|l|}{ CC16 } \\
\hline Concentration $\mu \mathrm{g} \cdot \mathrm{L}^{-1}$ & $8.6(7.1-13.1)$ & $9.7(7.1-12)$ & $9.7(6.9-13.3)$ & $8.6(7-10.5)$ & 0.90 \\
\hline Ratio CC16 to SP-D & $0.12(0.06-0.18)$ & $0.12(0.09-0.21)$ & $0.12(0.08-0.19)$ & $0.11(0.08-0.17)$ & 0.21 \\
\hline SP-D concentration $\mu \mathrm{g} \cdot \mathrm{L}^{-1}$ & $87(55-112)$ & $63(50-96)$ & $80(53-110)$ & $75(59-111)$ & 0.13 \\
\hline Creatinine $\mathrm{mg} \cdot \mathrm{L}^{-1}$ & $0.91(0.81-0.99)$ & $0.84(0.8-0.94)$ & $0.96(0.82-1.05)$ & $0.89(0.81-0.97)$ & 0.62 \\
\hline Urea $\mathrm{mg} \cdot \mathrm{L}^{-1}$ & $243(204-301)$ & 262 (212-288) & 271 (244-295) & $242(186-257)$ & 0.11 \\
\hline
\end{tabular}

Data are presented as median (interquartile range), unless otherwise stated. CC16: Clara cell protein; NO: nitric oxide; SP: surfactant protein. ${ }^{\#}:<5$ cigarettes $\cdot$ day ${ }^{-1}$;

$\because: \geqslant 5$ cigarettes day ${ }^{-1}$. Statistical analysis: one-way ANOVA followed by Dunnett's post hoc test. *: $p<0.05$ compared with nonsmokers.

absolute amount of recovered CC16 or by adjusting the CC16 concentration for the plasma/NALF urea concentration ratio. Notably, passive smokers showed a systemic tendency to lower CC16 levels in NALF compared with nonsmokers, especially after adjustment for the plasma/NALF urea concentration ratio (Dunnett's post hoc test $\mathrm{p}=0.08$ ). The most statistically significant difference, however, concerned the concentration of exhaled NO, which was noticeably reduced in the group of heavy smokers (median $42 \%$ lower than in nonsmokers). Concentrations of albumin in NALF and of CC16 and SP-D in serum, in contrast, did not vary between the different groups.

Multivariate analyses confirmed the decrease of NALF CC16 (normalised to urea ratio; slope $-0.76, p=0.02$ ) and of exhaled NO (slope $-0.38, \mathrm{p}=0.04$ ) in the group of heavy smokers and the lack of change in the other markers measured in NALF or serum. Figures 1 and 2 illustrate the changes in the covariateadjusted concentrations of CC16 in NALF, of exhaled NO, and
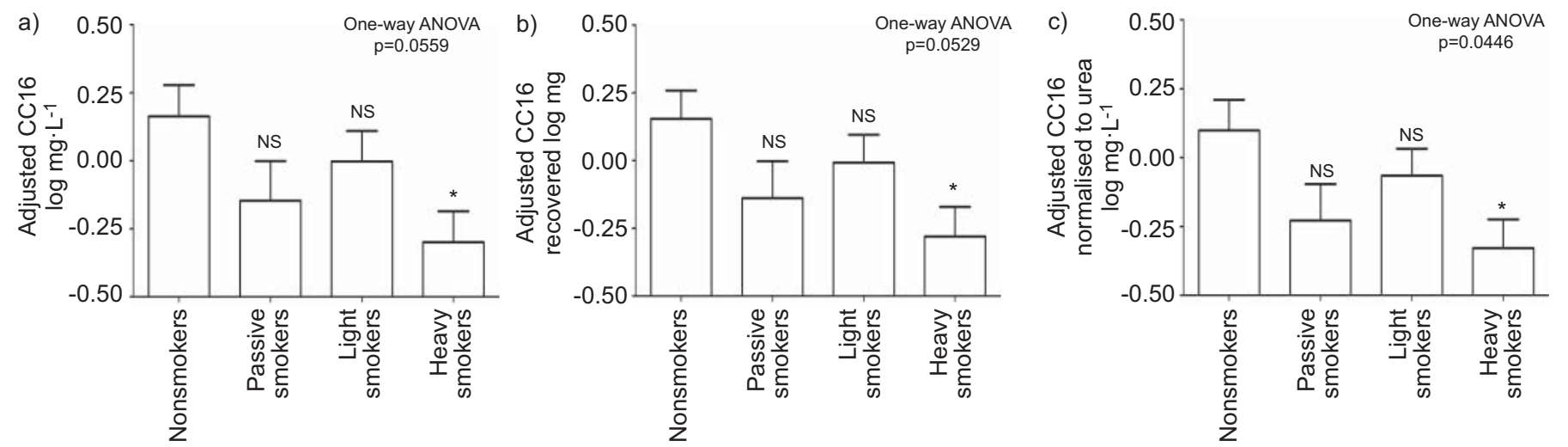

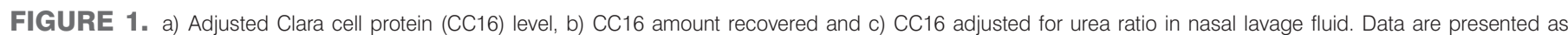

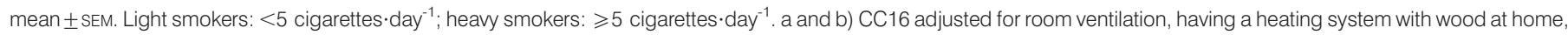

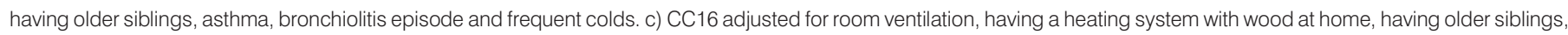
asthma and frequent colds. Statistical analysis: one-way ANOVA followed by Dunnett's post hoc test. NS: nonsignificant; *: p<0.05 compared with nonsmokers. 

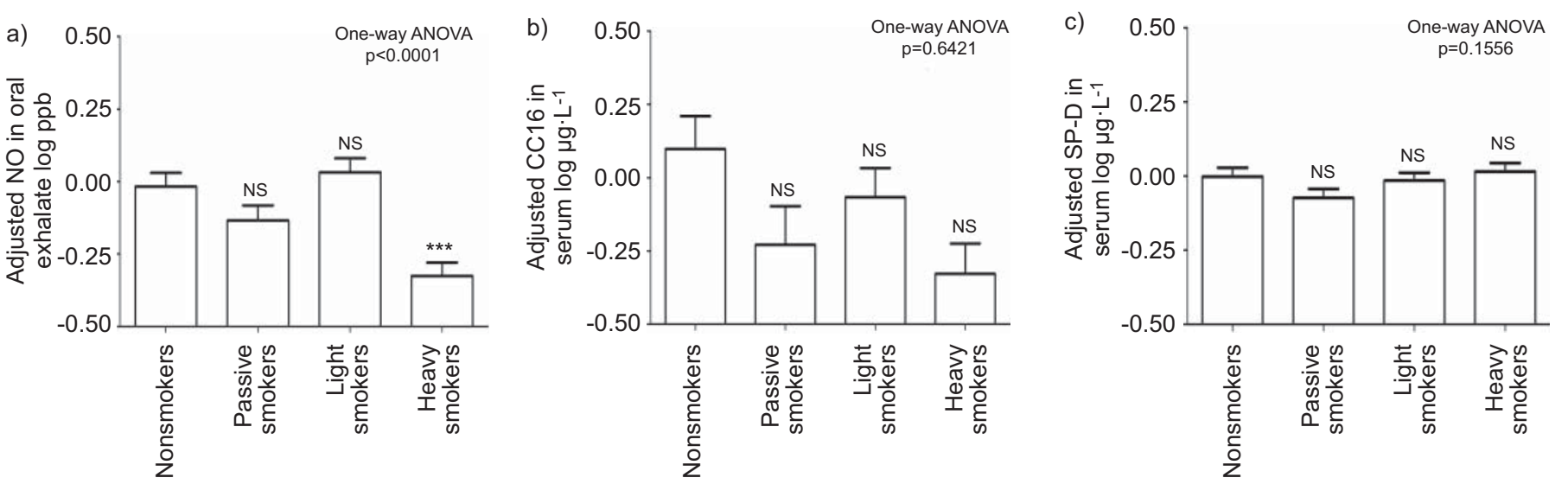

FIGURE 2. a) Adjusted nitric oxide (NO) levels in oral exhalate, b) adjusted Clara cell protein (CC16) levels in serum and c) adjusted surfactant protein (SP)-D levels in serum. Data are presented as mean \pm SEM. Light smokers: $<5$ cigarettes.day ${ }^{-1}$; heavy smokers: $\geqslant 5$ cigarettes.day ${ }^{-1}$. a) NO adjusted for use of air fresheners, having a heating system with wood at home, allergic rhinitis, bronchitis episode, frequent colds, immunoglobulin (Ig)E against aeroallergens and total IgE in serum. b) CC16 adjusted for age, body mass index, living in a rural area, use of bleach as a cleaning agent at home, having a heating system with wood, having older siblings, cumulated chlorinated pool attendance, asthma, bronchiolitis episode, time of sample collection and serum creatinine. c) SP-D adjusted for sex, day care attendance, parent with higher education, maternal smoking during pregnancy, room ventilation, use of bleach as a cleaning agent at home, having a heating system with wood, having a furred pet, asthma, frequent colds, hay fever, IgE against aeroallergens and total IgE in serum. Statistical analysis: one-way ANOVA followed by Dunnett's post hoc test. NS: nonsignificant; ***: $p<0.001$ compared with nonsmokers.

of CC16 and SP-D in serum. We also examined whether the decreased levels of exhaled NO and of CC16 in NALF in smokers correlated with the smoking history. There was a highly significant negative association between exhaled $\mathrm{NO}$ and the number of cigarettes smoked per day (fig. 3a). A negative association also emerged between CC16 in NALF and cigarette consumption but without reaching the level of statistical significance (fig. 3b). Similar associations were observed with the number of pack-years (results not shown). There was no apparent relationship between CC16 in NALF and exhaled NO, as evidenced by a Spearman correlation value of 0.16 and a $\mathrm{p}$-value of 0.28 . There were also no differences in the covariateadjusted levels of CC16 and of exhaled NO between adolescents with or without respiratory symptoms (results not shown). No statistically significant interaction between smoking status and sex was detected in the tested models. As illustrated in figure 3, exhaled NO and CC16 in NALF decreased rather similarly in males and females (exhaled NO: females $r=-0.54, p=0.0001$, males $r=-0.50, p=0.008$; CC16 in NALF: females $r=-0.26$, $\mathrm{p}=0.16$, males $\mathrm{r}=-0.16, \mathrm{p}=0.43)$.

\section{DISCUSSION}

The concentrations of CC16 in NALF and of NO in exhaled air were decreased in adolescents smoking $\geqslant 5$ cigarettes $\cdot$ day $^{-1}$. The low levels of these biomarkers persisted after adjustment for potential confounders and were correlated with the cigarette consumption. Quite remarkably, these biomarker changes occurred at a level of cumulative smoking that was about one order of magnitude lower than reported so far in adult smokers [15]. We found no change in the serum levels of CC16 and SP-D, which suggests that the deep lung epithelium had not yet been affected at this stage of smoking. This is really no surprise given the very low cumulative smoking of our adolescent study participants in comparison with previous studies reporting altered serum levels of these lung biomarkers $[12,13,27,28]$.
None of the studied biomarkers showed significant changes among adolescents who were light smokers or passive smokers, although there was a tendency of CC16 in NALF to decrease with passive smoking. This lack of biomarker response to passive smoking might appear inconsistent with the wealth of data, including those in the present study, showing that environmental tobacco smoke increases the risks of respiratory symptoms and other ailments [29]. The explanation for this discrepancy probably lies in the fact that studied biomarkers reflect chronic damage to the airways, which might require much higher cumulative doses of smoking than do respiratory symptoms. This is probably the reason why we could not detect any association between biomarker levels and respiratory symptoms in any of the groups exposed to cigarette smoke.

Our findings necessarily raise the question of the pathological significance of these early decreases of exhaled $\mathrm{NO}$ and of CC16 in NALF of smoking adolescents. Regarding exhaled NO, KHARITONOV et al. [15] have suggested that the reduced levels of this biomarker in smokers probably result from changes occurring in the upper respiratory tract. The authors proposed several mechanisms by which cigarette smoke might lower NO concentrations in exhaled breath: a downregulation of the $\mathrm{NO}$ synthase due to the NO present in the cigarette smoke itself, an inhibitory effect of $\mathrm{CO}$ which is also present in cigarette smoke, or else an inhibition of bacterial growth [15]. Whatever the exact mechanism, it is important to consider that NO plays an important role in the respiratory tract, in particular in the regulation of pulmonary and airway blood flow and the nonspecific defence mechanisms [30]. This suggests that a chronic reduction of such a mediator is likely to have detrimental effects. The cigarette smoke-induced changes in exhaled NO were only observed in the group of heavy smokers and not in the groups of light smokers or passive smokers, meaning that these effects require a certain smoke dose to be elicited. A similar conclusion was drawn by GABRIELE et al. [16] when they found decreased 

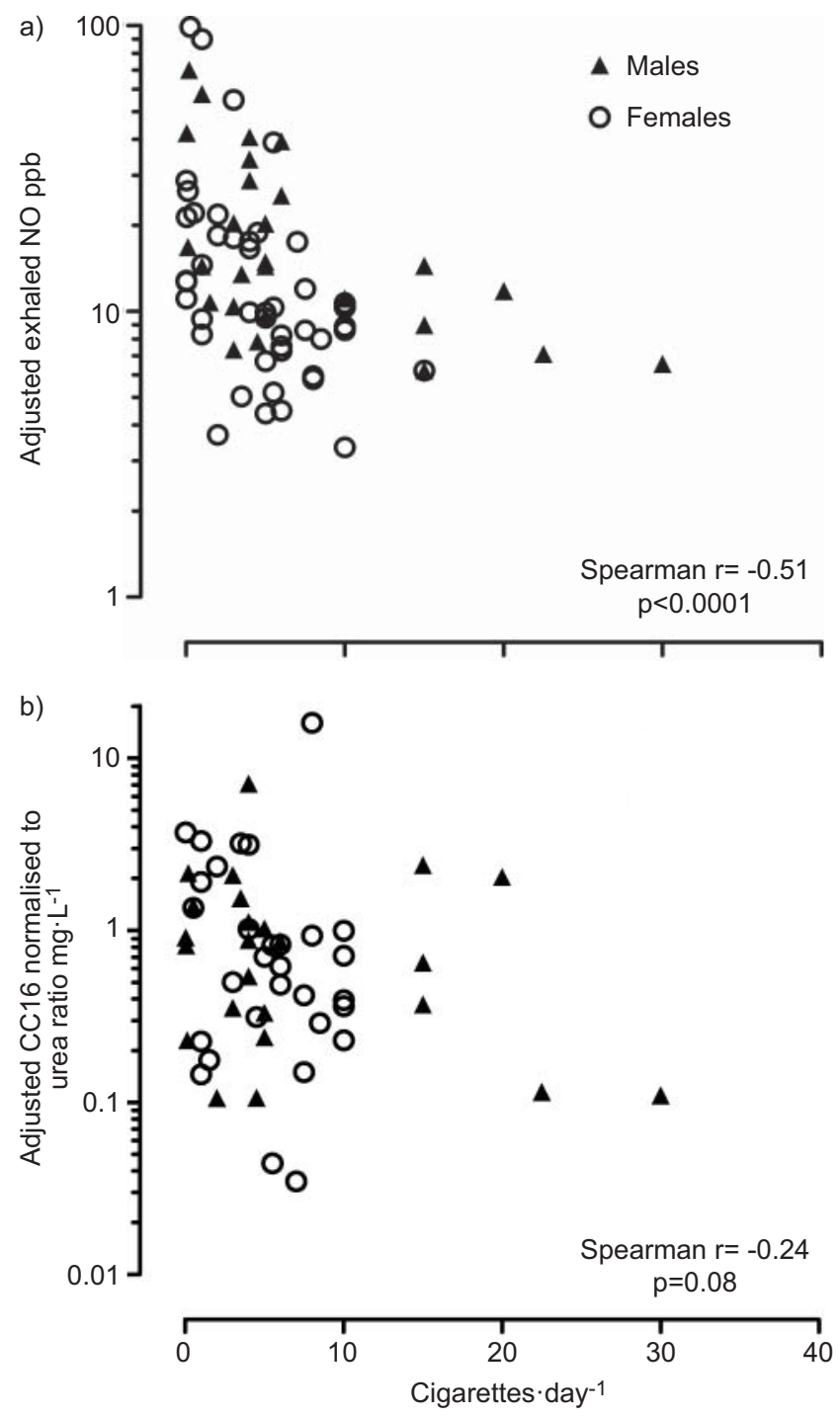

FIGURE 3. Relationships between daily cigarette consumption and epithelial biomarkers in males and females. a) Nitric oxide (NO) in exhaled air, b) Clara cell protein (CC16) normalised to urea in nasal lavage fluid. a) NO adjusted for use of air fresheners, having a heating system with wood at home, allergic rhinitis, bronchitis episode, frequent colds, immunoglobulin (Ig)E against aeroallergens and total lgE in serum. b) CC16 adjusted for room ventilation, having a heating system with wood at home, having older siblings, asthma and frequent colds. Relationships (both sexes together) were assessed using the Spearman nonparametric correlation test.

exhaled NO levels in infants exposed pre- and post-natally to tobacco smoke but not in never-exposed infants or in infants exposed to tobacco smoke only after birth.

It has been known a long time that the Clara cell is a sensitive target of cigarette smoke. Several studies have shown that cigarette smoking reduces the number of Clara cells and thereby the concentrations of CC16 in bronchoalveolar lavage, induced sputum and serum. CC16 is also a mediator that appears to have important regulatory and anti-inflammatory functions in the respiratory tract [4]. Moreover, Clara cells have the capability to differentiate into mucus-secreting cells [31], thus contributing to the evolution towards COPD stage 0, i.e. "at risk" according to the Global Initiative for Chronic Obstructive Lung Disease
(GOLD) [32]. Even if the exact role of CC16 at the level of the nasal cavity has not been elucidated, it appears reasonable to assume that cigarette smoke can cause similar epithelial damage in the upper airways to that in the lower airways. This possibility is supported, for instance, by the study of HADAR et al. [33], which describes goblet cell hyperplasia and thicker epithelium in the nasal mucosa of smokers.

A possible limitation of our study concerns the assessment of cigarette smoke exposure. Findings reported here were made in an add-on to a study initially designed to assess the impact of the environment on the health of adolescents. Although exposure to tobacco smoke was an important risk factor considered in our study, we did not measure exposure biomarkers such as serum or urinary cotinine, which anyway reflect only the recent exposure to tobacco smoke. However, to achieve the most objective assessment possible, we used the smoking habits reported by the adolescents themselves during a personal interview rather than those provided in the questionnaire filled by the parents. The fact that the main study was not focused on the effect of smoking might be more an advantage than an inconvenience. Our participants were blinded to the tested hypothesis, which allowed us to minimise the participation and response biases, which are important in this type of investigation. The reliability of our data about smoking habits is supported by the very significant dose-response relationships that emerged between exhaled NO and the daily or cumulative cigarette consumption.

In conclusion, the current biomarker study on adolescents shows that tobacco smoke can cause early changes in the airways as evidenced by decreased levels of CC16 in NALF and of NO in exhaled air. Quite remarkably, these effects are already apparent at cumulative cigarette consumption levels well below 1 pack-yr. These airway alterations in adolescents starting to smoke refute the common belief that tobacco smoke causes adverse effects occurring only later during adult life.

\section{SUPPORT STATEMENT}

This study was supported by the Belgian Science Policy (Brussels, Belgium) ANIMO (Indoor risk factors for childhood respiratory diseases: development and application of noninvasive biomarkers). A. Bernard is Research Director of the National Fund for Scientific Research (Brussels).

\section{STATEMENT OF INTEREST}

A statement of interest for E. Van Miert can be found at www.erj. ersjournals.com/site/misc/statements.xhtml

\section{REFERENCES}

1 van Beek EJ, Hoffman EA. Functional imaging: CT and MRI. Clin Chest Med 2008; 29: 195-216.

2 Kharitonov SA, Barnes PJ. Biomarkers of some pulmonary diseases in exhaled breath. Biomarkers 2002; 7: 1-32.

3 Quirce $S$, Lemière $C$, Blay $F$, et al. Noninvasive methods for assessment of airway inflammation in occupational settings. Allergy 2010; 65: 445-458.

4 Hermans C, Bernard A. Lung epithelium-specific proteins: characteristics and potential applications as markers. Am J Respir Crit Care Med 1999; 159: 646-678.

5 Irvin CG. The nose: a window into the asthmatic lung? Clin Exp Allergy 2010; 40: 839-840.

6 Kharitonov SA, Barnes PJ. Clinical aspects of exhaled nitric oxide. Eur Respir J 2000; 16: 781-792. 
7 Sin DD, Leung R, Gan WQ, et al. Circulating surfactant protein D as a potential lung-specific biomarker of health outcomes in COPD: a pilot study. BMC Pulm Med 2007; 7: 13.

8 Braido F, Riccio AM, Guerra L, et al. Clara cell 16 protein in COPD sputum: a marker of small airways damage? Respir Med 2007; 101: 2119-2124.

9 The Health Consequences of Smoking: A Report of the Surgeon General. Atlanta, Centers for Disease Control and Prevention, 2004

10 ASPECT (Analysis of the Science and Policy for European Control of Tobacco) Consortium. Tobacco or Health in the European Union: Past, Present and Future. Luxembourg, European Commission, 2004 http:/ / ec.europa.eu/health/ph_determinants/life_style/Tobacco/ Documents/tobacco_fr_en.pdf

11 Kjaergaard T, Cvancarova M, Steinsvaag SK. Smoker's nose: structural and functional characteristics. Laryngoscope 2010; 120: 1475-1480.

12 Robin M, Dong P, Hermans C, et al. Serum levels of CC16, SP-A and SP-B reflect tobacco-smoke exposure in asymptomatic subjects. Eur Respir J 2002; 20: 1152-1161.

13 Dell Ommo M, Hermans C, Muzi G, et al. Serum Clara cell protein (CC16) in healthy young smokers. Biomarkers 2000; 5: 158-164.

14 Shijubo N, Itoh $Y$, Yamaguchi T, et al. Serum and BAL Clara cell 10 $\mathrm{kDa}$ protein (CC10) levels and CC10-positive bronchiolar cells are decreased in smokers. Eur Respir J 1997; 10: 1108-1114.

15 Kharitonov SA, Robbins RA, Yates D, et al. Acute and chronic effects of cigarette smoking on exhaled nitric oxide. Am J Respir Crit Care Med 1995; 152: 609-612.

16 Gabriele C, Asgarali R, Jaddoe VW, et al. Smoke exposure, airway symptoms and exhaled nitric oxide in infants: the Generation $\mathrm{R}$ study. Eur Respir J 2008; 32: 307-313.

17 Lederer DJ, Enright PL, Kawut SM, et al. Cigarette smoking is associated with subclinical parenchymal lung disease: the MultiEthnic Study of Atherosclerosis (MESA)-lung study. Am J Respir Crit Care Med 2009; 180: 407-414.

18 Bernard A, Carbonnelle S, Dumont X, et al. Infant swimming practice, pulmonary epithelium integrity, and the risk of allergic and respiratory diseases later in childhood. Paediatrics 2007; 119: 1095-1103.

19 American Thoracic Society, European Respiratory Society. ATS/ ERS recommendations for standardized procedures for the online and offline measurement of exhaled lower respiratory nitric oxide and nasal nitric oxide, 2005. Am J Respir Crit Care Med 2005; 171: 912-930.

20 Hermans C, Bernard A. Pneumoproteinaemia: a new perspective in the assessment of lung disorders. Eur Respir J 1998; 11: 801-803.

21 Cavaliere F, Schiavello R, Masieri S, et al. Urea to calculate the dilution of nasal secretum collected by lavage. Acta Otorhinolaryngol Belg 1986; 40: 542-547.

22 Kaulbach HC, White MV, Igarashi Y, et al. Estimation of nasal epithelial lining fluid using urea as a marker. J Allergy Clin Immunol 1993; 92: 457-465.

23 Aragon TJ. Medical Epidemiology. "epitools" - R package for epidemiologic data and graphics. 2008. www.medepi.com/epitools

24 R Development Core Team. R: A Language and Environment for Statistical Computing. Vienna, Institute for Statistics and Mathematics, 2008. www.r-project.org

25 Wickham H. ggplot2: elegant graphics for data analysis. New York, Springer, 2009. http:/ / had.co.nz/ggplot2/book

26 Moravcsik MJ. Citation context classification of a citation classic concerning citation context classification. Soc Stud Sci 1988; 18: 515-521.

27 Broeckaert F, Clippe A, Knoops B, et al. Clara cell secretory protein (CC16): features as a peripheral lung biomarker. Ann NY Acad Sci 2000; 923: 68-77.

28 Honda Y, Takahashi H, Kuroki Y, et al. Decreased contents of surfactant proteins A and D in BAL fluids of healthy smokers. Chest 1996; 109: 1006-1009.

29 Lovasi GS, Diez Roux AV, Hoffman EA, et al. Association of environmental tobacco smoke exposure in childhood with early emphysema in adulthood among nonsmokers: the MESA-lung study. Am J Epidemiol 2010; 171: 54-62.

30 Barnes PJ, Belvisi MG. Nitric oxide and lung disease. Thorax 1993; 48: 1034-1043.

31 Nadel JA. Role of epidermal growth factor receptor activation in regulating mucin synthesis. Respir Res 2001; 2: 85-89.

32 Pauwels RA, Buist AS, Calverley PM, et al. Global strategy for the diagnosis, management, and prevention of chronic obstructive pulmonary disease. NHLBI/WHO Global Initiative for Chronic Obstructive Lung Disease (GOLD). Am J Respir Crit Care Med 2001; 163: 1256-1276.

33 Hadar T, Yaniv E, Shvili Y, et al. Histopathological changes of the nasal mucosa induced by smoking. Inhal Toxicol 2009; 21: 1119-1122. 